\title{
Oral Rehabilitation With Dental Implants for Teeth Involved in a Maxillary Fibrous Dysplasia
}

\author{
Alberto Monje, ${ }^{*}$ Florencio Monje, ${ }^{\dagger}$ Fernando Suarez, ${ }^{*}$ Raúl González-García, ${ }^{\dagger}$ Laura Villanueva-Alcojol, ${ }^{\dagger}$ and Hom-Lay Wang ${ }^{*}$
}
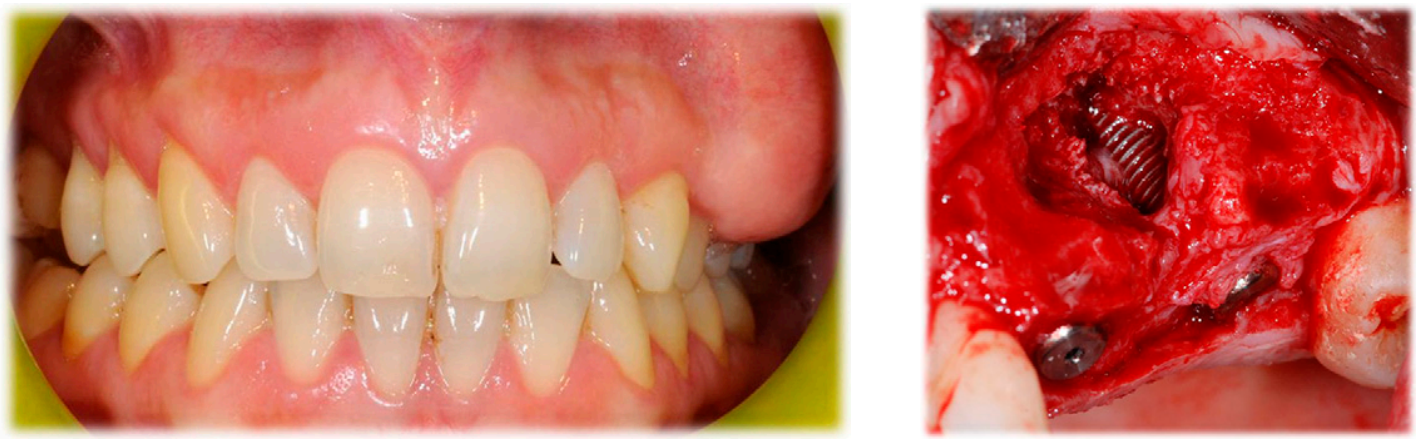

Introduction: Fibrous dysplasia (FD) is a rare but important benign lesion affecting the maxillofacial region that presents bone pain, deformities, and fracture. Although FD presents radiographic patterns, it has to be analyzed histologically for a final diagnosis. Currently, there is no universally accepted guide for FD treatment. This case report describes a successful case of excision of the entire lesion in the upper left maxilla and the placement of two dental implants for oral rehabilitation, followed by guided bone regeneration.

Case Presentation: A 49-year-old female patient presented with a growth on the left side of the maxilla that developed 4 years previously. Cone-beam computed tomography was used to analyze the lesion and clinical features, and the FD was confirmed by histologic analysis. Extraction of the teeth involved with the lesion and excising the lesion was suggested. This was followed by two-implant placement for oral rehabilitation, grafting the cavity with an osteoconductive material. Both implants were successfully integrated, as confirmed by clinical examination and a resonance frequency test.

Conclusion: Dental implant and related guided bone regeneration can be successfully obtained in a patient with an FD lesion. Clin Adv Periodontics 2013;3:208-213.

Key Words: Bone transplantation; dental implants; fibrous dysplasia of bone; maxillofacial abnormalities; pathology, oral.

\section{Background}

Fibro-osseous lesions are characterized by replacement of normal bone by fibrous tissue containing a newly formed mineralized product. Fibrous dysplasia (FD) and ossifying fibroma are the most common fibro-osseous lesions, which may be associated with functional and esthetic complications. ${ }^{1}$ These lesions usually present a diagnostic dilemma, as diagnosis must be based on clinical evidence, histologic analysis, and radiographic features. FD, however, creates radiographic patterns that are virtually indistinguishable from other lesions affecting the bones. ${ }^{2}$

FD is a rare but important benign lesion affecting the maxillofacial region that presents bone pain, deformities, and fractures. FD is a genetically based disease with mutations

\footnotetext{
* Department of Periodontics and Oral Medicine, University of Michigan School of Dentistry, Ann Arbor, MI.

† Department of Oral and Maxillofacial Surgery, Center of Implantology, Oral and Maxillofacial Surgery, Badajoz, Spain.
}

Submitted February 16, 2012; accepted for publication March 24, 2012

doi: 10.1902/cap.2012.120015 in the gene (GNSA I) encoding the $\alpha$-subunit of stimulatory G-protein $\left(G \alpha_{s}\right)$ that results in the increased production of cyclic adenosine monophosphate, affecting the proliferation and differentiation of preosteoblast. ${ }^{3}$ The clinical severity of the lesion depends on the time of appearance of the mutation. ${ }^{3}$ FD lesions are defined as "monostotic" and "polyostotic" depending on the number of bones involved. The monostotic form accounts for $70 \%$ to $85 \%$ of cases and does not progress into a polyostotic form. ${ }^{4}$ There is a slight female predilection, usually presenting in the first three decades of life. ${ }^{5}$ The right unilateral nature of FD is noted in almost all the cases reported, with the maxilla and frontal bones the most commonly involved. . $^{2,5}$

Radiologic features of FD include a poorly defined fusiform enlargement especially in the mandible or generalized enlargement of the maxilla. The margins of the lesion, "ground-glass" appearance, and displacement of maxillary sinus are some other features related to maxillary FD lesions. ${ }^{2}$ Although these features can be localized in a panoramic radiograph, computed tomography is often required for a more complete evaluation for the diagnosis and to evaluate treatment alternatives. 
The increased rate of bone resorption in FD has classically been treated by bisphosphonates. However, bone refilling the area where the lesion appeared has been observed in only $\approx 50 \%$ of the defects. ${ }^{6}$ Hence, the most predictable treatment, although more aggressive, is the excision of the entire affected area and grafting it with bone grafts. Although autologous bone has been considered the "gold standard" because of its osteogenic, osteoconductive, and osteoinductive abilities, morbidity of the donor area, resorption, and cost have led to the use of bone substitutes. Refilling the cavity facilitates future placement of endosseous implants if the patient wants to restore the original dentition function. The use of endosseous implants has been well established with a good long-term success. ${ }^{7}$ However, to our knowledge, the outcome of dental implants has not been described in patients with fibro-osseous lesions repaired with an osseous substitute. This paper reports successfully rehabilitated dental implants in a patient with monostotic FD in the upper maxilla.

\section{Clinical Presentation}

A 49-year-old white female presented to the Center of Implantology, Oral and Maxillofacial Surgery, Badajoz, Spain, in December 2009 with pain in the left second upper molar (tooth \#8) and an asymmetric growth on the left side of the maxilla that started to increase in size 4 years ago (Fig. 1). She was a non-smoker and otherwise healthy. Intraoral examination revealed good oral health and a high level of hygiene. After clinical exploration, it was decided that the mass on the left side of the maxilla was probably an osseous exostosis due to the long-term asymptomatic history of the lesion. However, we decided to explore the lesion with cone-beam computed tomography (CBCT), which revealed a $19 \times 20 \mathrm{~mm}$ well-defined ground-glass lesion. The patient was informed that a biopsy was needed to determine the final diagnosis and written informed cosent was obtained. Four weeks later, an incisional biopsy was done under intravenous sedation and local anesthesia at the mass level in the left maxilla and a core obtained for the histologic evaluation (Fig. 2). The evaluation confirmed a FD lesion. Using microscopy, trabeculae of woven bone in a background of fibrous tissue was observed. Radiographically, it was observed that the lesion was in close contact with the roots of the left

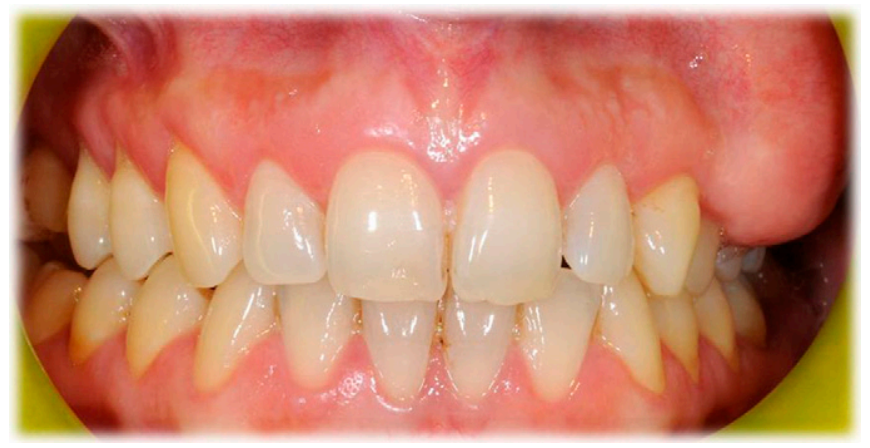

FIGURE 1 Frontal view of the initial growth.

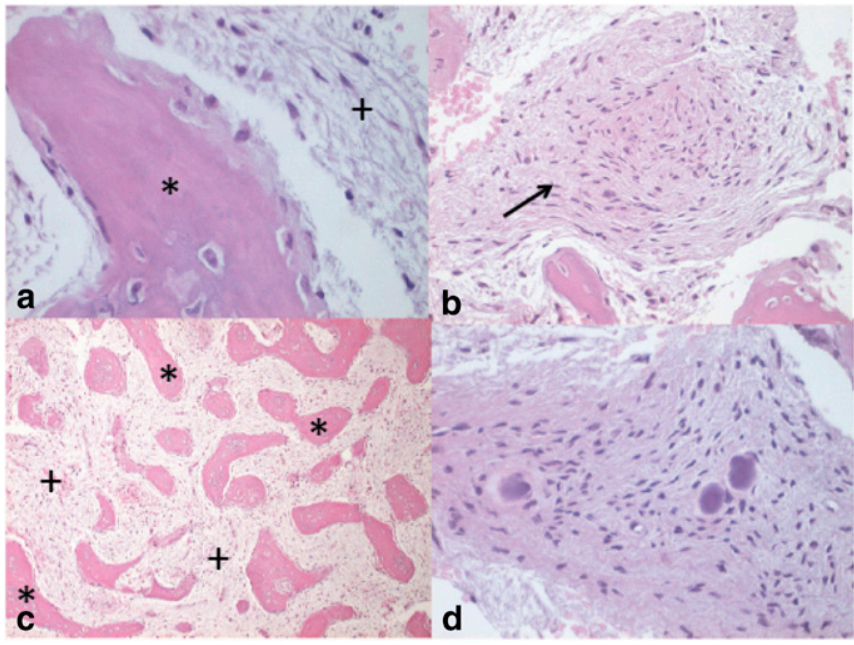

FIGURE 2 Histologic aspect of the lesion. 2a Microscopic detail of the relationship between trabecular bone $\left(^{*}\right)$ and stroma $(+)$. Hematoxylin \& eosin; magnification $\times 400$. $2 \mathrm{~b}$ Area of stroma showing more fibrous density (arrow). Hematoxylin \& eosin; magnification $\times 200.2 c$ Immature trabecular bone (") divided by fibrous tissue (+). Hematoxylin \& eosin; magnification $\times 100$. 2d Detail of the loose fibrillar stroma with fibroblastic cells. Hematoxylin \& eosin; magnification $\times 400$.

first and second upper premolars (teeth \#12 and \#13) and left first upper molar (tooth \#14) (Figs. 3 and 4). For these reasons, 2 months later, the involved teeth were extracted and the lesion excised by curettage (Fig. 5).

\section{Case Management}

After a healing period of 10 months, oral rehabilitation was needed in the left maxillae area. Under intravenous sedation and local anesthesia, two $4 \times 15$-mm implants ${ }^{\S}$ were placed (Fig. 6). This was followed by filling the remaining cavity with a bone substitute material" because of its osteoconductive abilities. A membrane was placed to promote the osseous regeneration. Implant stability quotient (ISQ) was measured using an implant stability meter\# just before wound closure. The ISQ for both implants were measured immediately after placement and 6 months later. These were 81 and 66 and 81 and 70, respectively. A ceramicmetal screw-retained fixed prostheses was delivered after conventional loading 6 months after implant placement.

\section{Clinical Outcomes}

One year later, no sign of inflammation, peri-implant bone loss, or mobility were reported and, although there was no microscopic evidence of osseointegration, stability of implants was achieved for oral reconstruction/rehabilitation (Figs. 7 and 8).

\footnotetext{
‡ i-CAT, Imaging Sciences International, Hatfield, PA.

NobelSpeedy and NobelGroovy implants, Nobel Biocare, Gothenburg, Sweden.

" Bio-Oss, Geistlich, Wolhusen, Switzerland.

- Bio-Gide, Geistlich.

\# Osstell, Gothenburg, Sweden.
} 


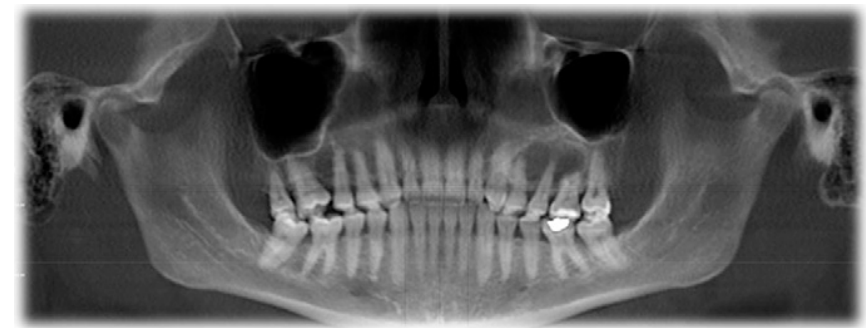

FIGURE 3 Panoramic radiograph showing the extent of the involvement.

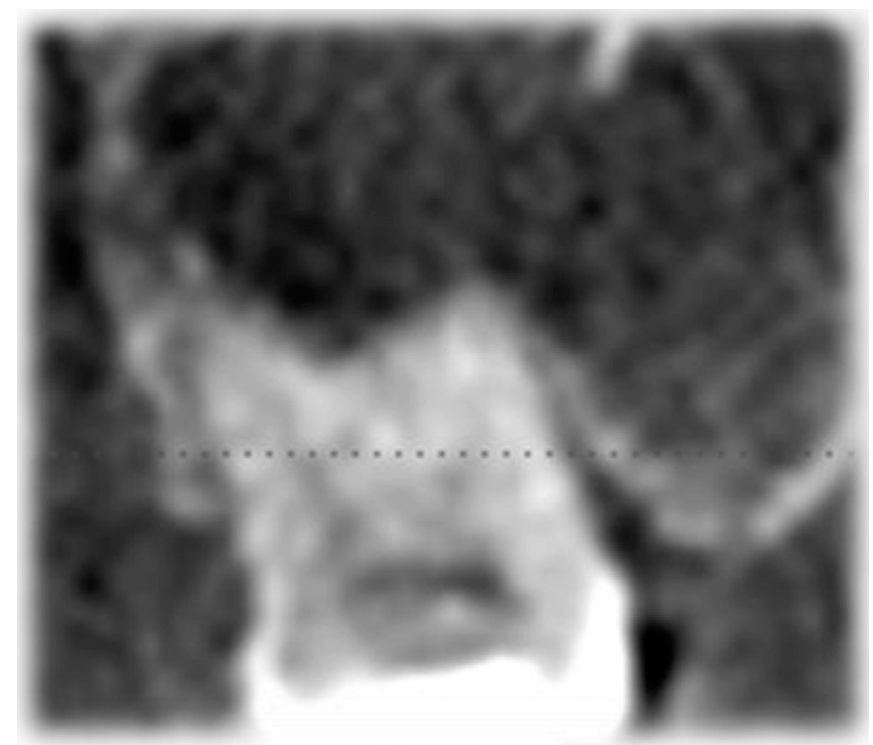

FIGURE 4 Sagittal view of the FD in close contact with a tooth of the left upper maxilla.

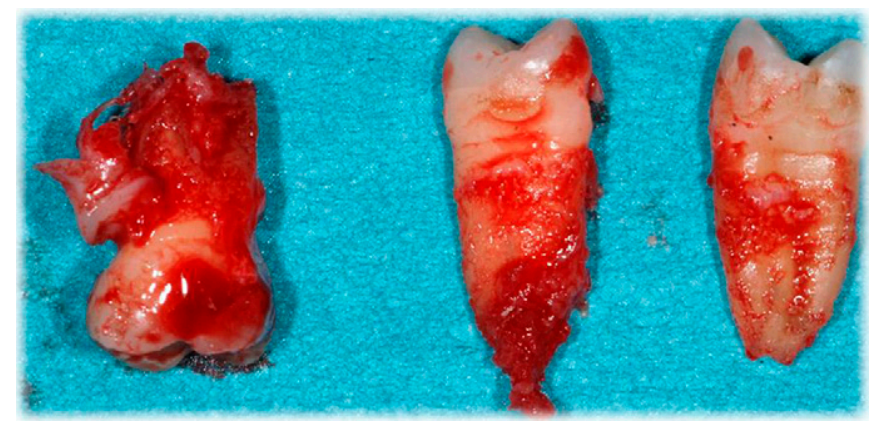

FIGURE 5 Teeth after extraction with rest of the lesion on their roots.

\section{Discussion}

Osseointegration is defined as the close contact between bone and implant material in histologic observations and, in clinical terms, as the ankylosis of the implant in bone. ${ }^{8}$ However, in some clinical situations, bone grafting procedures are required before placement of dental implants. Bone augmentation allows implant treatment that might otherwise not be an option. High survival rates have been obtained in implants placed after bone augmentation procedures. ${ }^{9}$ In the present case, a patient with a monostotic FD in the left side of the maxilla was successfully managed by the excision of the whole lesion, grafting the defect with a bone substitute, and placing two dental implants with implant stability and no clinically detectable mobility.

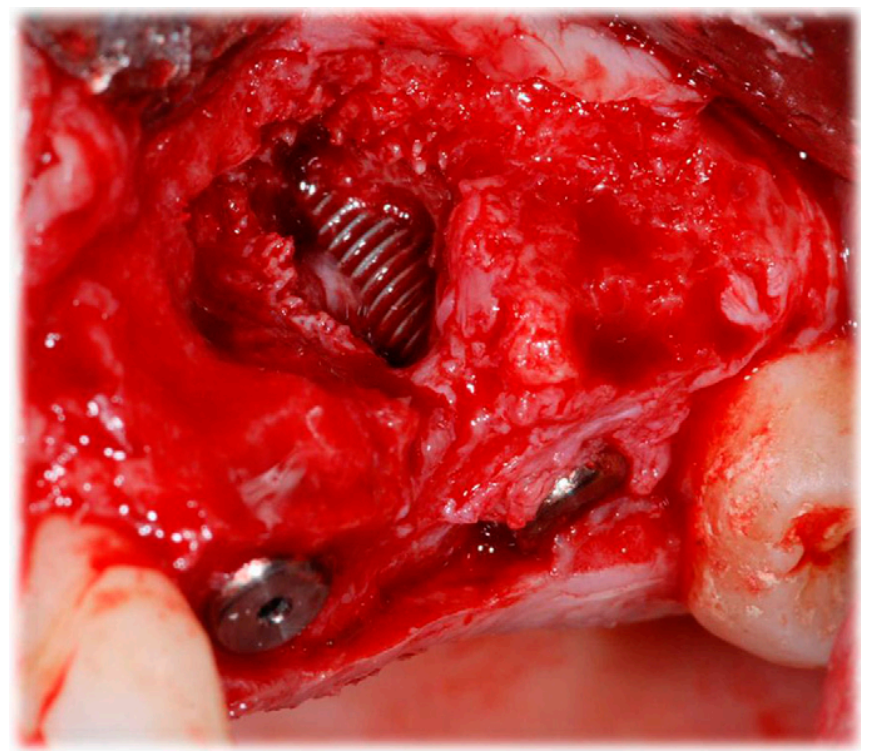

FIGURE 6 Implant placement 10 months after the excision of the lesion.

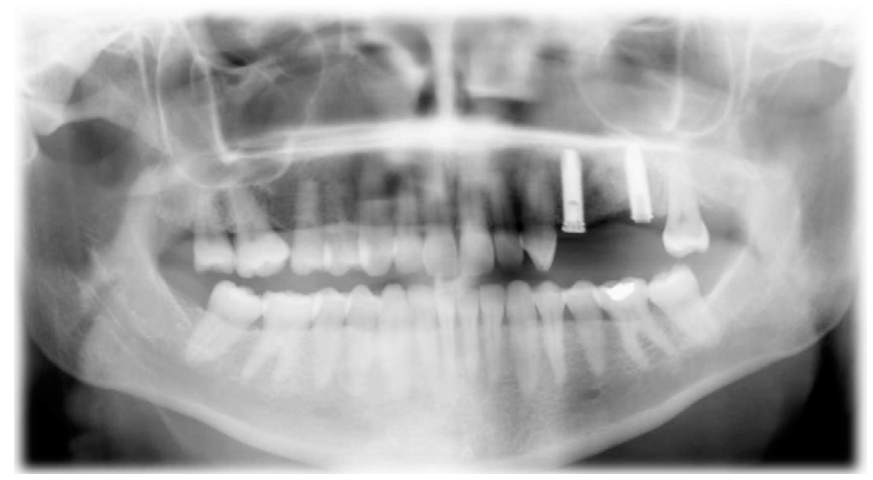

FIGURE 7 Frontal view of both implants 12 months after implant placement.

FD is a benign fibro-osseous lesion that accounts for nearly $7 \%$ of all benign tumors of bone, although, generally speaking, most experts believe that it should not be classified as a true bone neoplasm. ${ }^{10}$ This lesion is an hamartomatous condition related to mutations in gene (GNSA I) encoding $G \alpha_{\mathrm{s}}$ that results in the increased production of cyclic adenosine monophosphate, affecting the proliferation and differentiation of preosteoblast. $^{3}$ FD was initially described in 1891 by Recklinghausen and named in 1938 by Lichtenstein. ${ }^{10}$ In this disease, normal bone is replaced with a disorganized fibrous connective tissue and woven bone, which is generally a painless process and is often found by a routine clinical or radiologic examination. ${ }^{10}$ Our finding was a result of the patient's concerns about the growth in the maxilla area and the pain related to the teeth closest to the lesion.

FD must be diagnosed by complete clinical, radiographic, and histologic studies. This frequently requires a differentiation with ossifying fibroma. Ossifying fibroma is a lesion of bone, as well, but is a monofocal process that tends to grow centrifugally and be demarcated. ${ }^{10}$

Clinically, the disease is classified as monostotic or polyostotic. The monostotic form, as in our finding, is isolated 


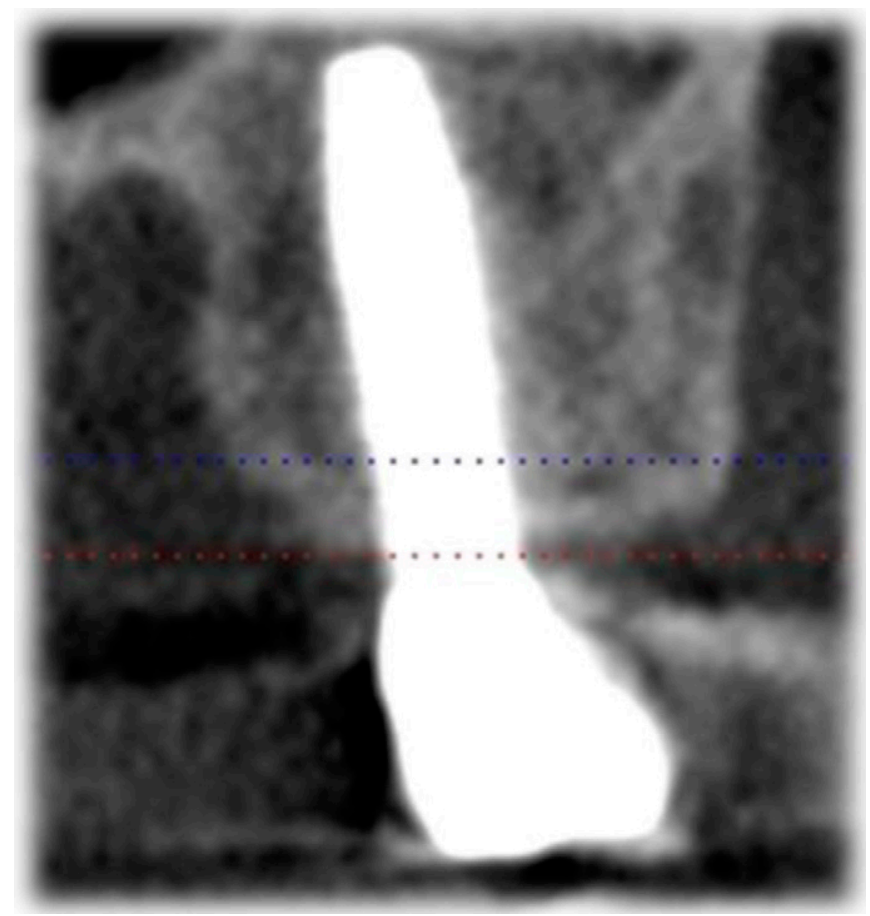

FIGURE 8 Sagittal view of one of the implants 12 months after implant placement.

to a single bone, whereas the polyostotic form involves $>1$ non-contiguous bone. ${ }^{4}$ The monostotic form is not a precursor of the polyostotic form and accounts for $70 \%$ to $85 \%$ of FD cases. ${ }^{4}$ When the bones of the face are involved, the lesion often includes contiguous bones, such as maxilla, sphenoid, zygoma, and ethmoid bones. In the jaws, FD is more frequently found in the maxilla than in the mandible, mainly in the posterior region. ${ }^{4}$

CBCT provides comprehensive information for the diagnosis of a lesion in the maxillofacial area. CBCT can be used to determine the extent, specific dimension, and radiodensity of FD. ${ }^{5}$ It also allows for virtual implant therapy planning with special three-dimension software programs. Furthermore, the emission of radiation of the CBCT is $25 \%$ less than a panoramic radiography and 40 to 60 times less than a classic CT scan. ${ }^{11}$

The three most common radiologic appearances of FD in the craniofacial skeleton are the pagetoid, sclerotic, and cystic patterns. These are seen in the CBCT as bone expansion and scattered islands of bone formation in a low-attenuation field, an homogeneous appearance with a ground-glass appearance, and a well-defined low-attenuation lesion with a sclerotic margin referred to as an "eggshell lesion." 2 The use of CBCT in the present case was required to plan our approach and final treatment for oral rehabilitation with dental implants. Threedimensional radiographic exploration must be performed after healing to confirm the absence of reactivation of the FD.
Currently, there is no universally accepted guideline for FD treatment. Those cases in which the lesion is asymptomatic and does not cause deformities or functional impairment should simply be monitored. ${ }^{4}$ A surgical approach is required only when anatomic structures are in danger of being compressed or the function or esthetics of the patient is compromised. However, even when a complete excision is performed, lifelong monitoring is often recommended because of the potential for late growth, dysfunction, and small risk of sarcomatous change. ${ }^{10}$ Recurrence of the disease is uncommon in adults but is common during growth. A 6-year follow-up study showed that almost all of the surgically treated patients presented satisfactory outcomes (only one of 13 presented recurrence of FD). ${ }^{12}$ In the present case, implant placement was delayed for two main reasons: 1 ) a deficient amount of bone to accommodate proper placement; and 2) the necessity of following up the lesion to ensure that there was no recurrence. It is important to point out that a shortterm follow-up of this specific case cannot draw any definitive conclusion regarding long-term predictability. Studies with a longer follow-up and larger sample size are needed to validate the findings reported here. When surgery is not indicated, relief of bone pain and reduction of osteoclastic activity with partial filling of osteolytic lesions can be achieved with bisphosphonates therapy. ${ }^{6}$ In this case, we completely excised the lesion, filled the void with a bone substitute to promote osseous regeneration, placed two dental implants, and then restored the area with an implant-supported prostheses.

Cheung et al. ${ }^{13}$ reported a successful bone-titanium screw contact in patients with FD. Although the contact was higher in normal bone than in dysplasic bone, it was not statistically significant. Thus, they suggested that longer screws be used to compensate for the reduced bone contact percentage. Bajwa et al. ${ }^{14}$ reported a case of oral rehabilitation using endosseous implants in both maxilla and mandible in a patient with craniofacial FD. After 5 years of follow-up, although microscopic examination was unable to prove osseointegration, a direct functional connection between dysplasic bone and the surface of a load-bearing implant was evidenced by the lack of symptoms, soft-tissue inflammation, and peri-implant bone loss. ${ }^{14}$ Uckan et al. ${ }^{15}$ preferred to treat their patient with a total maxillectomy and then place implants into the normal zygomatic bone to facilitate future oral rehabilitation. To the best of our knowledge, this case report is one of the first attempts using combination approaches (excise the lesion, augment lesion defect, place dental implants, and restore with implant-supported prostheses) to restore the patient back to original function and esthetics. 


\section{Summary}

Why is this case new information? management of this case?
What are the keys to successful

- To the best of our knowledge, this is the first case to document the success of dental implant osseointegration in a patient with FD.

- Proper diagnosis (e.g., CBCT and histologic confirmation) and complete debridement of FD lesion

- Good oral hygiene

- Carefully executed guided bone regeneration and wound closure, abided by the "PASS" principle ${ }^{16}$ for guided bone regeneration

- Obtain implant primary stability at the time of implant placement and avoid any premature loading during healing

What are the primary limitations to success in this case?
- Wrong diagnosis

- Poorly executed lesion debridement, implant surgery, and bone augmentation

- Poor oral hygiene and premature implant loading

\section{Acknowledgments}

This work was supported by the Foundation for the Study of Implantology, Oral and Maxillofacial Surgery (FEDICOM), Badajoz, Spain. The authors thank Dr. Ramón Gómez de Tejada, Pathology Service of Infanta Cristina Hospital, Badajoz, Spain, for the histologic analysis and Dr. Miguel Padial-Molina, Department of Periodontics and Oral Medicine, University of Michigan, Ann Arbor, Michigan, for describing and helping us understand the histologic aspect of the lesion. The authors report no conflicts of interest related to this case report.
CORRESPONDENCE:

Dr. Alberto Monje, Juan Miró 16-17, 06011 Badajoz, Spain. E-mail: amonjec@umich.edu. 


\section{References}

1. Toyosawa S, Yuki M, Kishino M, et al. Ossifying fibroma vs fibrous dysplasia of the jaw: Molecular and immunological characterization. Mod Pathol 2007;20:389-396.

2. Sontakke SA, Karjodkar FR, Umarji HR. Computed tomographic features of fibrous dysplasia of maxillofacial region. Imaging Sci Dent 2011;41:23-28.

3. Cohen MM Jr, Howell RE. Etiology of fibrous dysplasia and McCuneAlbright syndrome. Int J Oral Maxillofac Surg 1999;28:366-371.

4. Lustig LR, Holliday MJ, McCarthy EF, Nager GT. Fibrous dysplasia involving the skull base and temporal bone. Arch Otolaryngol Head Neck Surg 2001;127:1239-1247.

5. MacDonald-Jankowski D. Fibrous dysplasia in the jaws of a HongKong population: Radiographic presentation and systematic review. Dentomaxillofac Radiol 1999;28:195-202.

6. Chapurlat RD, Hugueny P, Delmas PD, Meunier PJ. Treatment of fibrous dysplasia of bone with intravenous pamidronate: Long-term effectiveness and evaluation of predictors of response to treatment. Bone 2004;35:235-242.

7. Buser D, Mericske-Stern R, Bernard JP, et al. Long-term evaluation of non-submerged ITI implants. Part 1: 8-year life table analysis of a prospective multi-center study with 2359 implants. Clin Oral Implants Res 1997;8:161-172.

8. Albrektsson T, Zarb GA. Current interpretations of the osseointegrated response: Clinical significance. Int J Prosthodont 1993;6:95-105.
9. Hämmerle $\mathrm{CH}$, Jung $\mathrm{RE}$, Feloutzis A. A systematic review of the survival of implants in bone sites augmented with barrier membranes (guided bone regeneration) in partially edentulous patients. J Clin Periodontol 2002;29(Suppl. 3):226-231; discussion 232-233.

10. Kim DD, Ghali GE, Wright JM, Edwards SP. Surgical treatment of giant fibrous dysplasia of the mandible with concomitant craniofacial involvement. J Oral Maxillofac Surg 2012;70:102-118.

11. Sukovic P. Cone beam computed tomography in craniofacial imaging. Orthod Craniofac Res 2003;6(Suppl. 1):31-36, discussion 179-182.

12. Keijser LC, Van Tienen TG, Schreuder HW, Lemmens JA, Pruszczynski M, Veth RP. Fibrous dysplasia of bone: Management and outcome of 20 cases. J Surg Oncol 2001;76:157-166; discussion 167-168.

13. Cheung LK, Samman N, Pang M, Tideman H. Titanium miniplate fixation for osteotomies in facial fibrous dysplasia - A histologic study of the screw/bone interface. Int J Oral Maxillofac Surg 1995;24:401-405.

14. Bajwa MS, Ethunandan M, Flood TR. Oral rehabilitation with endosseous implants in a patient with fibrous dysplasia (McCune-Albright syndrome): A case report. J Oral Maxillofac Surg 2008;66:2605-2608.

15. Uckan S, Oguz Y, Uyar Y, Ozyesil A. Reconstruction of a total maxillectomy defect with a zygomatic implant-retained obturator. J Craniofac Surg 2005;16:485-489.

16. Wang HL, Boyapati L. "PASS" principles for predictable bone regeneration. Implant Dent 2006;15:8-17.

indicates key references. 